\title{
Spontaneous rectus sheath hematoma as a rare cause of abdominal pain in a patient under oral anticoagulation
}

\begin{abstract}
Rectus sheath haematoma (RSH) is a known complication of abdominal wall trauma, surgery and excessive strain of abdominal wall. It can also occur spontaneously in patients under oral anticoagulation. The condition has a wide-spectrum of severity depending on its size, aetiology and development of complications. RSH usually runs a benign course but it can present with hypovolemic shock or abdominal compartment syndrome. Treatment is usually conservative and outcome is generally favourable. Here we report a case of a spontaneous rectus sheath haematoma secondary to oral anticoagulation therapy with warfarin sodium.
\end{abstract}

Keywords: rectus sheath hematoma, Warfarin, abdominal pain
Volume I Issue 4 - 2014

\author{
Mahmut Yesin,' Macit Kalçık,' Mustafa Ozan \\ Gürsoy,' Süleyman Karakoyun, ${ }^{2}$ Mehmet \\ Özkan ${ }^{1,2}$ \\ 'Department of Cardiology, Kosuyolu Kartal Heart Training and \\ Research Hospital, Turkey \\ ${ }^{2}$ Department of Cardiology, Kars Kafkas University, Faculty of \\ Medicine, Turkey
}

\begin{abstract}
Correspondence: Mahmut Yesin, Department of Cardiology, Kosuyolu Kartal Heart Training and Research Hospital, Denizer Cad. Cevizli, Kvs. No:2 Cevizli Kartal /lstanbul, Turkey, Tel
\end{abstract} 902165001599, Email mahmutyesin@yahoo.com

Received: July 23, 2014 | Published: September 13, 2014

\section{Introduction}

Rectus sheath hematoma (RSH) is a rare entity ${ }^{1}$ which is caused by rupture of the perforating branches of the inferior epigastric artery running in the preperitoneal fat, causing a large haematoma spreading in the looser rectus sheath space. Non-invasive imaging modalities including CT scan are the key to establish its early diagnosis. It is often misdiagnosed as many other serious intra-abdominal diseases, such as appendicitis, diverticulitis, cholecystitis, incarcerated inguinal hernia, torsion of an ovarian cyst or acute pancreatitis. ${ }^{2} \mathrm{RSH}$ is usually seen in patients with coagulation disorders and other predisposing causes including abdominal trauma, previous surgery, asthma, stretching, hypertension, pregnancy, intraabdominal injection, and iatrogenic causation during laparoscopy. Patients with rectus sheath hematoma usually present with severe abdominal pain and abdominal wall mass. Our patient who received warfarin therapy for 18years due to atrial fibrillation presented to our emergency department with abdominal pain.

\section{Case report}

A 67-year-old woman was referred to our emergency department with abdominal pain. She was under warfarin therapy for atrial fibrillation. Physical examination revealed weakness, severe tenderness and rebound tenderness in the lower abdomen. A mass was felt in the right side of the abdomen. She had no family history of bleeding diathesis or hematological diseases. Her blood pressure was $100 / 50 \mathrm{mmHg}$ and electrocardiogram showed atrial fibrillation. The patient's laboratory findings were as follows: haemoglobin 7,1g/dl, leukocytes $7800 / \mu \mathrm{L}$, international normalized ratio 2.7 . Her platelet count $(226 \times 103 / \mu \mathrm{L})$, liver function tests, urea, creatinin and serum amylase were within normal limits. There was not any predisposing condition (constipation, coughing etc.) that might lead to development of RSH except for being under oral anticoagulation; she did not use any antiplatelet agent beside warfarin sodium. An abdominopelvic computed tomography (CT) scan was performed which showed a large right-sided rectus sheath haematoma (Figure 1). She was diagnosed with rectus sheath hematoma, and anticoagulant treatment was discontinued. Three units of packed cell were transfused and patient was closely monitored for hemodynamic status. Her symptom disappeared in the following days, and rectus sheath hematoma was spontaneously resolved within 15 days.

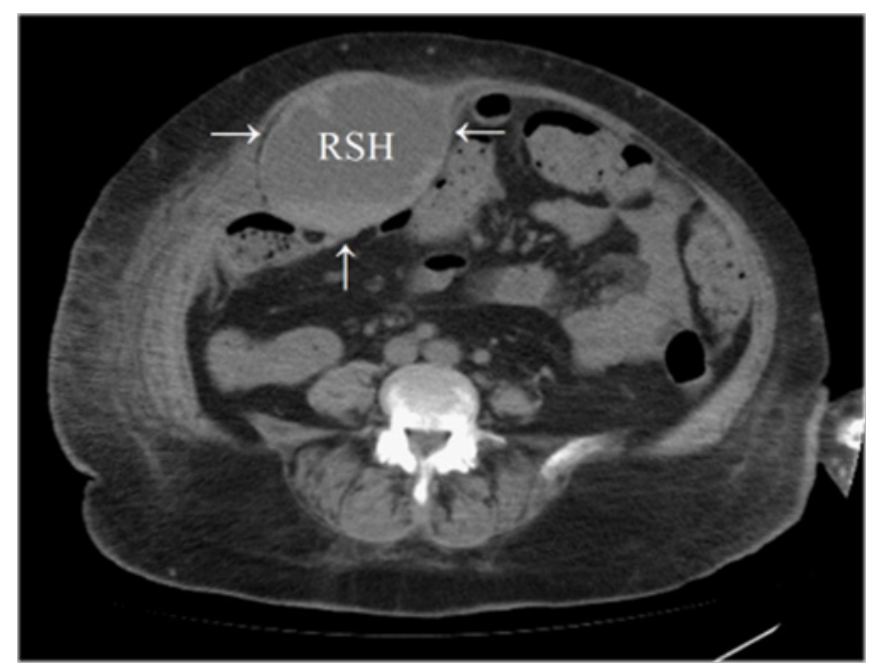

Figure I Abdominopelvic computed tomography scan without contrast shows a huge right rectus sheath hematoma (RSH).

\section{Discussion}

RSH is an uncommon and frequently misdiagnosed cause of abdominal pain. It is usually seen in right lower abdomen. There are many risk factors including increased age, anticoagulant therapy, platelet disorders, trauma, recent surgery, injection procedures, and physical exercise as well as increased intraabdominal pressure from 
coughing, sneezing, vomiting, or straining during urination, defecation or labor. ${ }^{3}$ The diagnosis of rectus sheath hematoma is conducted thorough sonography or CT scan but CT scan is the choice procedure. Berna et al. ${ }^{4}$ classified RSH into three types using CT findings:

1) Grade I is an intramuscular hematoma with an observable increase in muscle size.

2) Grade II is also an intramuscular hematoma but with blood between the muscle and transversalis fascia.

3) Grade III hematoma may or may not affect the muscle and blood is seen between the transversalis fascia and muscle in the peritoneum and prevesical space that results in a drop in hemoglobin. ${ }^{4}$

Clinical manifestations include abdominal pain (84\%), Fothergill sign (an abdominal mass that does not cross the midline and remains palpable when the rectus muscles are flexed), a substantial drop in hemoglobin, abdominal wall ecchymosis (present in $21 \%$ of hematomas). ${ }^{5}$ Treatment of RSH is usually conservative which includes analgesia, fluid resuscitation, blood transfusion and treatment of the underlying condition in hemodynamically stable cases. Surgical management is usually performed in an emergency setting in patients with hypovolemic shock or abdominal compartment syndrome. Surgery consists of exploration of the rectus sheath and lack of bleeding control is associated with high mortaliy. Although coil embolisation technique is time-consuming, it is expensive, not always available, and should be considered in high risk cases. ${ }^{6}$ In brief, awareness of this rare clinical condition is important in the differential diagnosis of acute abdominal pain. RSH should be suspected in patients on anticoagulation therapy, who present with clinical manifestations of acute abdomen. Failure to recognize this condition could result in unnecessary laparotomy since the majority of patients with rectus sheath hematoma can be managed conservatively.

\section{Acknowledgement}

None.

\section{Conflicts of interest}

Authors declare that there is no conflict of interest.

\section{Funding}

None.

\section{References}

1. De Martino C, Martino A, Giamattei RM, et al. Spontaneous rectus sheath hematoma: a rare condition with uneasy diagnosis and multidisciplinary treatment. Ann Ital Chir. 2011;82(5):399-404.

2. Denard PJ, Fetter JC, Zacharski LR. Rectus sheath hematoma complicating low molecular weight heparin therapy. Int J Lab Hematol. 2007;29(3):190-194.

3. Moreno Gallego A, Aguayo JL, Flores B, et al. Ultrasonography and computed tomography reduce unnecessary surgery in abdominal rectus sheath haematoma. Br J Surg. 1977;84(9):1295-1297.

4. Berna JD, Garcia Medina V, Guirao J, et al. Rectus sheath hematoma: diagnostic classification by CT. Abdom Imaging. 1966;21(1):62-64.

5. Cherry WB, Mueller PS. Rectus sheath hematoma: review of 126 cases at a single institution. Medicine (Baltimore). 2006;85(2):105-110.

6. Salemis NS, Gourgiotis S, Karalis G. Diagnostic evaluation and management of patients with rectus sheath hematoma. A retrospective study. Int J Surg. 2010;8(4): 290-293. 